Pacific

Journal of

Mathematics

UBIQUITY OF GEOMETRIC FINITENESS IN MAPPING CLASS GROUPS OF HAKEN 3-MANIFOLDS

Sungbok Hong and Darryl McCullough 


\title{
UBIQUITY OF GEOMETRIC FINITENESS IN MAPPING CLASS GROUPS OF HAKEN 3-MANIFOLDS
}

\author{
Sungbok Hong And Darryl McCullough
}

\begin{abstract}
For a Haken 3-manifold $M$ with incompressible boundary, we prove that the mapping class group $\mathcal{M}$ acts properly discontinuously on a contractible simplicial complex, with compact quotient. This implies that every torsionfree subgroup of finite index in $\mathcal{M}$ is geometrically finite. Also, a simplified proof of the fact that torsionfree subgroups of finite index in $\mathcal{M}$ exist is given. All results are given for mapping class groups that preserve a boundary pattern in the sense of K. Johannson. As an application, we show that if $F$ is a nonempty compact 2-manifold in $\partial M$ such that $\partial M-F$ is incompressible, then the classifying space $\mathrm{BD}$ iff $(M$ rel $F)$ of the diffeomorphism group of $M$ relative to $F$ has the homotopy type of a finite aspherical complex.
\end{abstract}

\section{Introduction.}

The mapping class group $\mathcal{H}(F)$ of a 2-manifold is the group of isotopy classes of diffeomorphisms, $\pi_{0}(\operatorname{Diff}(F))$. As is well-known, it acts properly discontinuously on a Teichmüller space, which is topologically a Euclidean space (traditionally only the action of the orientation-preserving classes was considered). This classical setup was refined by Harer $[\mathbf{8}, \mathbf{9}]$, who found an ideal triangulation of Teichmüller space for which $\mathcal{H}(F)$ acts simplicially, then constructed a contractible simplicial complex in the first barycentric subdivision of this triangulation which is invariant and has finite quotient under the action. Consequently, if $\Gamma$ is any torsionfree subgroup of finite index in $\mathcal{H}(F)$ (and such subgroups always exist), the quotient of the action of $\Gamma$ on this complex is a finite $K(\Gamma, 1)$-complex. That is, $\Gamma$ is a geometrically finite group.

For a compact 3-manifold $M$, a great deal is known about $\mathcal{H}(M)$ (see for example $[\mathbf{2 0}]$ or the surveys $[\mathbf{2 5}, \mathbf{2 7}])$. In the present paper, we extend the aforementioned property of torsionfree subgroups of $\mathcal{H}(F)$ to the case of Haken 3-manifolds. To postpone the introduction of boundary patterns, we state here only a corollary of our main theorem. In the corollary, $\mathcal{H}(M$ rel $W)$ means $\pi_{0}(\operatorname{Diff}(M$ rel $W))$, where $\operatorname{Diff}(M$ rel $W)$ is the group of diffeomorphisms of $M$ which restrict to the identity on $W$. 
Corollary 7.10. Let $M$ be a Haken 3 -manifold and let $W$ be a compact 2dimensional submanifold of $\partial M$ such that $\partial M-W$ is incompressible. Then any torsionfree subgroup of finite index in $\mathcal{H}(M$ rel $W)$ is geometrically finite.

In this result, $W$, or $\partial M-W$, or both may be empty. For Haken 3manifolds, it was already known that $\mathcal{H}(M$ rel $W)$ contains a finite-index subgroup which is geometrically finite [26], so the new information from the Main Theorem is that every torsionfree subgroup of finite index in $\mathcal{H}(M$ rel $W)$ is geometrically finite. It is a longstanding and apparently difficult open question whether a torsionfree finite extension of a geometrically finite group must be geometrically finite. Of course, if this were known, the result from [26] would imply our strengthened version.

Our method of proof is to construct a topological action of $\mathcal{H}(M$ rel $W)$ on a contractible simplicial complex (whose quotient is compact), and as we show in Section 3 this is sufficient to deduce the geometric finiteness of torsionfree finite-index subgroups. It would be interesting to give a more direct construction of a contractible complex, along the lines of those developed by Harer, admitting a simplicial action of $\mathcal{H}(M$ rel $W)$ with finite quotient.

The Kontsevich Conjecture (Problem 3.48 in the new version of R. Kirby's problem list [20]) asserts that the classifying space $\operatorname{BDiff}(M$ rel $\partial M)$ has the homotopy type of a finite complex when $M$ is a compact 3-manifold with nonempty boundary. This conjecture was recently proven for the case of Haken 3-manifolds in [14]. As observed there, the Kontsevich Conjecture for a Haken 3-manifold $M$ is equivalent to the assertion that $\mathcal{H}(M$ rel $\partial M)$ is geometrically finite. In Section 8 we use our results on geometric finiteness to deduce a generalization of the result from [14]:

Theorem 8.7. Let $M$ be a Haken 3-manifold with incompressible boundary, and let $F$ be a nonempty compact 2-manifold in $\partial M$ such that $\partial M-F$ is incompressible. Then $\operatorname{BDiff}(M$ rel $F)$ has the homotopy type of a finite complex.

The proof makes use of the extension of Nielsen's theorem to 3-manifolds made by Heil and Tollefson [15].

We will work in the context of 3-manifolds with boundary patterns. This lends greater generality to the results, and allows us to make direct use of Johannson's powerful characteristic submanifold theory for Haken manifolds. As is well-known, the characteristic submanifold was discovered and exploited independently by Johannson [17] and Jaco and Shalen [16]. We use Johannson's formulation because it is ideally suited to working with homotopy equivalences and homeomorphisms of 3-manifolds. In Section 2 we provide a brief exposition of the portion of Johannson's theory that we will use. In Section 3 we introduce a generalization of geometric finiteness, called (for lack of imagination) almost geometric finiteness. Any torsionfree 
finite-index subgroup of an almost geometrically finite group is geometrically finite. Using a theorem of Kamishima, Lee, and Raymond [18], we prove in Proposition 3.3 a key fact: An extension of a virtually finitely generated abelian group by an almost geometrically finite group is almost geometrically finite. The rest of the proof then follows the general approach of [26] to show that $\mathcal{H}(M)$ is an extension of this form, and hence is almost geometrically finite. It is necessary to work with a bit more precision than was needed in [26], since one can no longer evade difficulties by passing to subgroups of finite index in $\mathcal{H}(M)$. We also give a new proof that torsionfree subgroups of finite index in $\mathcal{H}(M)$ exist. This was proven in [26] by a very complicated argument; the new proof uses an algebraic fact from [28] to give a much shorter and more transparent proof. The final section contains the application to the Kontsevich Conjecture.

The authors thank BSRI-1422 and Nondirect Research Fund (K.R.F.) for support of their collaborative work, and acknowledge helpful discussions with Kyung-bai Lee and Leonard Rubin.

\section{Johannson's characteristic submanifold theory.}

We give here a brief review of the basic definitions of Johannson's formulation of the characteristic submanifold. We refer the reader to [17] for the original presentation, and also to Chapter 2 of [2] for a more extensive expository treatment with a number of examples.

A boundary pattern $\underline{\underline{m}}$ for an $n$-manifold $M$ is a finite set of compact, connected $(n-1)$-manifolds in $\partial M$, such that the intersection of any $i$ of them is empty or consists of $(n-i)$-manifolds. Thus when $n=3$, two elements of the boundary pattern intersect in a collection of arcs and circles, while if three elements meet, their intersection consists of a finite collection of points at which three intersection arcs meet. It is important in arguments to distinguish between elements of $\underline{\underline{m}}$ and the points of $M$ which lie in them, and we will always be precise in this distinction. The symbol $|\underline{\underline{m}}|$ will mean the set of points of $\partial M$ that lie in some element of $\underline{\underline{m}}$. When $|\underline{\underline{m}}|=\partial M, \underline{\underline{m}}$ is said to be complete. Provided that $\partial M$ is compact, we define the completion of $\underline{\underline{m}}$ to be the complete boundary pattern $\underline{\underline{m}}$ which is the union of $\underline{\underline{m}}$ and the set of components of the closure of $\partial \bar{M}-|\underline{\underline{m}}|$. In particular, the set of boundary components of $M$ is the completion of the empty boundary pattern $\underline{\underline{\emptyset}}$.

Suppose $(X, \underline{\underline{x}})$ is an admissibly imbedded codimension-zero submanifold of $(M, \underline{\underline{m}})$, which is admissibly imbedded in $(M, \underline{\underline{m}})$. The latter assumption guarantees that $X \cap \partial M=|\underline{\underline{x}}|$, that $|\underline{\underline{x}}|$ is contained in the topological interior of $|\underline{\underline{m}}|$ in $\partial M$, and that an element of $\underline{\underline{x}}$ which does not meet any other element of $\underline{\underline{x}}$ must be imbedded in the manifold interior of an element of $\underline{\underline{m}}$. Let $\underline{\underline{x}}^{\prime \prime}$ denote the collection of components of the frontier of $X$ in $M$. To 
split $M$ along $X$ means to construct the manifold with boundary pattern $\left(\overline{M-X}, \underline{\underline{\tilde{m}}} \cup \underline{\underline{x}}^{\prime \prime}\right)$, where the elements of $\underline{\underline{\tilde{m}}}$ are the closures of the components of $F-(X \cap F)$ for $F \in \underline{\underline{m}}$. The boundary pattern $\underline{\underline{\underline{m}}} \cup \underline{\underline{x}}^{\prime \prime}$ is called the proper boundary pattern on $\overline{M-X}$.

Maps which respect boundary pattern structures are called admissible. Precisely, a map $f$ from $(M, \underline{\underline{m}})$ to $(N, \underline{\underline{n}})$ is called admissible when $\underline{\underline{m}}$ is the disjoint union

$$
\underline{\underline{m}}=\coprod_{G \in \underline{\underline{n}}}\left\{\text { components of } f^{-1}(G)\right\} .
$$

An admissible map $h:(K, \underline{\underline{k}}) \rightarrow(X, \underline{\underline{x}})$, where $K$ is an arc or a circle and $(X, \underline{\underline{x}})$ is a 2- or 3-manifold, is called inessential if it is admissibly homotopic to a constant map (the constant map might not be admissible, but all the other maps in the homotopy must be admissible), otherwise it is called essential. A map $f:(X, \underline{\underline{x}}) \rightarrow(Y, \underline{y})$ between 2- or 3-manifolds (not necessarily of the same dimension) is called essential if for any essential path or loop $h:(K, \underline{\underline{k}}) \rightarrow(X, \underline{\underline{x}})$, the composition $f h:(K, \underline{\underline{k}}) \rightarrow(Y, \underline{\underline{y}})$ is essential. Notice that when $\underline{\underline{x}}$ is empty, this simply says that $f$ is injective on fundamental groups.

The group of admissible isotopy classes of admissible diffeomorphisms from $(M, \underline{\underline{m}})$ to $(M, \underline{\underline{m}})$ is denoted by $\mathcal{H}(M, \underline{\underline{m}})$. The classes that preserve the orientation of each component are indicated by a plus subscript, as in $\mathcal{H}_{+}(M, \underline{\underline{m}})$. The classes relative to the subset $\left|\underline{\underline{m_{1}}}\right|$, where $\underline{\underline{m_{1}}} \subseteq \underline{\underline{m}}$, are denoted by $\mathcal{H}\left(M, \underline{\underline{m}}\right.$ rel $\left.\left|\underline{\underline{m_{1}}}\right|\right)$. Suppose $\left.\langle h\rangle \in \mathcal{H} \overline{\overline{(M}}, \underline{\underline{m}}\right)$. Since $h^{-1}(|\underline{\underline{m}}|)=$ $|\underline{\underline{m}}|, h$ must carry each free side of $(M, \underline{\underline{m}})$ diffeomorphically to a free side of $(M, \underline{\underline{m}})$. Therefore $h$ is admissible for $(M, \underline{\underline{m}})$. Thus when working with mapping class groups of manifolds with boundary patterns, the requirement that the boundary pattern be complete is not at all restrictive.

An $i$-faced disc is a 2-disc whose boundary pattern is complete and has $i$ components. Observe that each element of $\underline{\underline{m}}$ is incompressible if and only if whenever $D$ is an admissibly imbedded 1 -faced disc in $(M, \underline{\underline{m}})$, the boundary of $D$ bounds a disc in $|\underline{\underline{m}}|$ which is contained in a single element of $\underline{\underline{m}}$. For most of Johannson's theory, a somewhat stronger condition is needed. The boundary pattern $\underline{\underline{m}}$ of a 3-manifold $M$ is called useful when the boundary of every admissibly imbedded $i$-faced disc in $(M, \underline{\underline{m}})$ with $i \leq 3$ bounds a disc

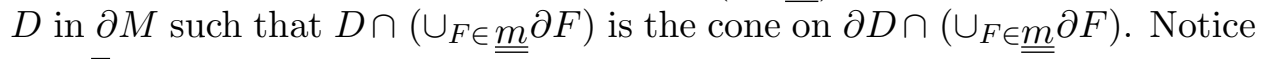
that $\underline{\underline{\emptyset}}$ is a useful boundary pattern on $M$ if and only $\partial M$ is incompressible.

A $\overline{\bar{S}}$ eifert fibering on a 3 -manifold $(V, \underline{\underline{v}})$ with boundary pattern is called an admissible Seifert fibering when the elements of $\underline{\underline{v}}$ are the preimages of the components of a boundary pattern of the orbit surface. Consequently the elements of $\underline{\underline{v}}$ must be tori or fibered annuli.

Assume that $\bar{V}$ carries a fixed structure as an $I$-bundle over $B$. Each component of the associated $\partial I$-bundle is a 2-manifold in $\partial V$, called a lid 
of the $I$-bundle. There are two lids when the bundle is a product, and one when it is twisted. Let $\underline{b}$ be a boundary pattern on $B$. The preimages of the elements of $\underline{\underline{b}}$ form a collection of squares and annuli in $\partial V$, called the sides of the $I$-bundle. The lid or lids, together with the sides, if any, form a boundary pattern $\underline{\underline{v}}$ on $V$. When $V$ carries this boundary pattern, the fibering is called an admissible $I$-fibering of $V$ as an $I$-bundle over $(B, \underline{b})$. We emphasize that for an admissible $I$-fibering, the lids are always elements of the boundary pattern.

We are now ready to introduce the characteristic submanifold. An admissible I-bundle or Seifert fibered space $(X, \underline{\underline{x}})$ in a 3 -manifold $(M, \underline{\underline{m}})$ is an $I$-bundle or Seifert fibered space imbedded in $M$ such that the inclusion defines admissible maps $(X, \underline{\underline{x}}) \rightarrow(M, \underline{\underline{m}})$ and $(X, \underline{\underline{x}}) \rightarrow(M, \underline{\underline{\underline{m}}})$. An admissible $I$-bundle or Seifert fibered space in $M$ is called essential when its frontier is an essential surface in $(M, \underline{\underline{m}})$. This implies that the inclusion of $(X, \underline{\underline{x}})$ into $(M, \underline{\underline{m}})$ is an essential map.

We suppose that $(M, \underline{\underline{m}})$ is a Haken 3-manifold with useful boundary pattern. A disjoint collection $(\Sigma, \underline{\underline{\sigma}})$ of essential admissible $I$-bundles and Seifert fibered spaces is a characteristic submanifold for $(M, \underline{\underline{m}})$ if

1) $(\Sigma, \underline{\underline{\sigma}})$ is full, i.e. the union of $\Sigma$ with any of the components of $\overline{M-\Sigma}$ is not a disjoint union of essential admissible $I$-bundles and Seifert fibered spaces,

2) (Engulfing property) any essential admissible $I$-bundle or Seifert fibered space $(X, \underline{\underline{x}})$ in $(M, \underline{\underline{m}})$ is admissibly isotopic into $(\Sigma, \underline{\underline{\sigma}})$, and

3) (Enclosing property) any essential map $f:(T, \underline{\underline{t}}) \rightarrow(M, \underline{\underline{m}})$ of a square, annulus, or torus into $(M, \underline{\underline{m}})$ is admissibly homotopic to a map with image in $\Sigma$.

One may combine Proposition 9.4, Corollaries 10.9 and 10.10 and Theorem 12.5 of $[\mathbf{1 7}]$ to see that every Haken 3-manifold with useful boundary pattern has a characteristic submanifold, and that the characteristic submanifold is unique up to admissible isotopy.

A Haken 3-manifold $(M, \underline{\underline{m}})$ whose completed boundary pattern $\underline{\underline{\underline{m}}}$ is useful is called simple if every component of the characteristic submanifold of $(M, \underline{\underline{\bar{m}}})$ is a regular neighborhood of an element of $\underline{\underline{\underline{m}}}$. When the boundary consists of tori, the interior of a simple 3-manifold admits a hyperbolic structure of finite volume. Then, by Mostow Rigidity and Waldhausen's theorem, $\mathcal{H}(M, \bar{\emptyset})$ is isomorphic to the group of isometries, hence is finite. One of the deep applications of Johannson's theory is the following generalization of this fact (although it was proven before the dissemination of Thurston's work on hyperbolic structures of 3-manifolds). It appears as Proposition 27.1 of [17]. 
Theorem 2.1 (Finite Mapping Class Group Theorem). Let $(M, \underline{\underline{m}})$ be a simple 3-manifold with complete and useful boundary pattern. Then $\mathcal{H}(M, \underline{\underline{m}})$ is finite.

\section{Almost geometric finiteness.}

We say that a group $G$ is almost geometrically finite if it acts smoothly and properly discontinuously on a contractible simplicial complex $L$, such that $L / G$ is compact. A subgroup of finite index in an almost geometrically finite group is almost geometrically finite. Almost geometrically finite groups are finitely generated (any group acting properly discontinuously on a connected locally compact space with compact quotient must be finitely generated). Every torsionfree subgroup of finite index in an almost geometrically finite group must be geometrically finite:

Lemma 3.1. Let $G$ be an almost geometrically finite group, and let $\Gamma$ be a torsionfree subgroup of finite index in $G$. Then $\Gamma$ is geometrically finite.

Proof. Let $G$ act properly discontinuously on the contractible complex $L$ with compact quotient. Since $\Gamma$ has finite index, $L / \Gamma$ is compact, and since $\Gamma$ is torsionfree, it acts freely on $L$. Therefore $L / \Gamma$ is locally an ANR and hence an ANR (see for example Theorem 5.4.5 of [32]). By [35], a finitedimensional compact ANR is homotopy equivalent to a finite simplicial complex, and therefore $\Gamma$ is geometrically finite.

We caution that in general, an almost geometrically finite group need not contain any geometrically finite subgroups of finite index: Schneebeli [30] constructed extensions $1 \rightarrow \mathbf{Z} / k \rightarrow E \rightarrow Q \rightarrow 1$ where $Q$ is geometrically finite but $E$ contains no torsionfree subgroups of finite index.

By Lemma 3.1, a group is geometrically finite if and only if it is almost geometrically finite and torsionfree. Simple examples of almost geometrically finite groups are finite groups (acting on a point) and finitely generated abelian groups (project to the quotient by the torsion subgroup and act on $\mathbf{R}^{n}$ by translations). By work of Borel and Serre [1], many arithmetic groups including $\mathrm{GL}(n, \mathbf{Z})$ are almost geometrically finite. Finitely generated virtually free groups are another interesting example; by [19] these are exactly the groups that act simplicially on trees with finite quotient and finite vertex stabilizers. In our context, mapping class groups of 2-manifolds are one of the most important examples:

Lemma 3.2. Let $B$ be a 2-manifold, not necessarily connected, of finite type with compact boundary, and let $\underline{\underline{b}}$ be a boundary pattern for $B$. Then $\mathcal{H}(B, \underline{\underline{b}})$ is almost geometrically finite.

Proof. Write $\underline{\underline{b}}=\underline{\underline{a}} \cup \underline{\underline{c}}$ where the elements of $\underline{\underline{a}}$ are arcs and those of $\underline{\underline{c}}$ are circles. Let $\bar{B}_{0}$ be obtained by removing all boundary circles that do not 
contain an element of $\underline{a}$. Let $P^{\prime}$ consist of one point from the interior of each element of $\underline{a}$ and let $P^{\prime \prime}$ consist of the points that are the intersection of two different elements of $\underline{a}$. It is not difficult to see that $\mathcal{H}(B, \underline{b})$ is isomorphic to a subgroup of finite index in $\mathcal{H}\left(B_{0}, P^{\prime} \cup P^{\prime \prime}\right)$. Thus we are reduced to proving the lemma for $\mathcal{H}(B, P)$, where $P$ is a finite subset of $\partial B$ that meets every boundary component of $B$.

We will first prove the lemma under the assumption that $B$ is connected. Suppose first that $\chi(B) \geq 0$. For $S^{2}$ with 0,1 , or 2 punctures, $\mathbf{R P}^{2}$ with 0 or 1 puncture, $D^{2}$ with 0 or 1 puncture, the Möbius band, or the Klein bottle, $\mathcal{H}(B, P)$ is finite, for the annulus it is virtually infinite cyclic, and for the torus it is $\mathrm{GL}(2, \mathbf{Z})$. From now on we assume that $\chi(B)<0$.

Suppose first that $P$ is empty, so $B$ is either closed or is a punctured closed surface. If $B$ is closed and orientable, then $\mathcal{H}(B)$ acts properly discontinuously on Teichmüller space. (Classically, from the viewpoint of conformal surfaces, only the action of the orientation-preserving classes was considered, but using the hyperbolic viewpoint as in [5], $\mathcal{H}(B)$ acts as well.) This action extends to the Harvey bordification $[\mathbf{1 0}, \mathbf{1 1}]$ of Teichmüller space, on which it acts smoothly with compact quotient. Lifting a smooth triangulation gives the necessary simplicial structure to conclude that $\mathcal{H}(B)$ is almost geometrically finite. If $B$ is orientable and punctured, then $\mathcal{H}(B)$ acts on the contractible complex $Y^{0}$ constructed by Harer [8, 9] with compact quotient. The analogues of these for nonorientable surfaces are given in Section 2 of [26]. Suppose now that $P$ is nonempty. Lemma 1.2 of [14] uses [13] to construct a contractible complex on which $\mathcal{H}(B, P)$ acts properly discontinuously.

We now assume that $B$ is not connected. Let $B_{1}, \ldots, B_{n}$ be the components of $B$, and let $P_{i}=P \cap B_{i}$. Assume first that each $\left(B_{i}, P_{i}\right)$ is diffeomorphic to $\left(B_{1}, P_{1}\right)$. From the connected case, there is a contractible simplicial complex $L$ such that $\mathcal{H}\left(B_{1}, P_{1}\right)$ acts properly discontinuously on $L$. Let $L_{i}$ be a copy of $L$ on which $\mathcal{H}\left(B_{i}, P_{i}\right)$ acts. Fix diffeomorphisms $f_{i}:\left(B_{1}, P_{1}\right) \rightarrow\left(B_{i}, P_{i}\right)$ and equivariant simplicial isomorphisms $\phi_{i}: L_{1} \rightarrow$ $L_{i}$. By means of these identifications, if $\langle h\rangle \in \mathcal{H}(B, P)$, and $h$ carries $B_{i}$ to $B_{j}$, we may regard $\langle h\rangle$ as carrying $L_{i}$ to $L_{j}$. For let $h_{i}: B_{i} \rightarrow B_{j}$ be the restriction of $h$ to $B_{i}$. Then let $h_{i}$ send $L_{i}$ to $L_{j}$ by $\phi_{j} \phi_{i}^{-1}$ 。 $\left\langle f_{i} f_{j}^{-1} h_{i}\right\rangle$. Now we define an action of $\mathcal{H}(B, P)$ on $\prod_{i=1}^{n} L_{i}$. Given $h$, define a permutation $\sigma$ by $h\left(B_{i}\right)=B_{\sigma(i)}$. Then, put $\langle h\rangle\left(x_{1}, \ldots, x_{n}\right)=$ $\left(\left\langle h_{\sigma^{-1}(1)}\right\rangle\left(x_{\sigma^{-1}(1)}\right), \ldots,\left\langle h_{\sigma^{-1}(n)}\right\rangle\left(x_{\sigma^{-1}(n)}\right)\right)$. This action is properly discontinuous with compact quotient, since the finite index subgroup of $\mathcal{H}(B, P)$ that preserves each component acts as a product of properly discontinuous actions each with compact quotient.

For the general case, partition the components of $(B, P)$ into maximal subsets such that each subset consists of pairwise diffeomorphic components. 
Let $F_{1}, \ldots, F_{m}$ be the unions of the components in the subsets, and let $Q_{i}=P \cap F_{i}$. By the previous paragraph, each $\mathcal{H}\left(F_{i}, Q_{i}\right)$ acts properly discontinuously on a contractible complex $K_{i}$. Since $\mathcal{H}(B, P)$ is the direct product of the $\mathcal{H}\left(F_{i}, Q_{i}\right)$, it acts properly discontinuously on the product of the $K_{i}$.

A useful observation is that if there is a surjective homomorphism $Q_{1} \rightarrow$ $Q_{2}$ with finite kernel, and $Q_{2}$ is almost geometrically finite, then $Q_{1}$ is also almost geometrically finite. In our proof that mapping class groups of Haken 3-manifolds are almost geometrically finite, we will use the following generalization of this observation, which is a direct consequence of a theorem of Kamishima, Lee, and Raymond [18]:

Proposition 3.3. Let $1 \rightarrow V \rightarrow G \rightarrow Q \rightarrow 1$ be an exact sequence of groups, such that $V$ contains a finitely generated abelian group of finite index and $Q$ is almost geometrically finite. Then $G$ is almost geometrically finite.

Proof. Consider a subgroup $\mathbf{Z}^{n}$ of finite index in $V$. Under conjugation by elements of $G$, its orbit consists of finitely many subgroups, whose intersection is a normal subgroup isomorphic to $\mathbf{Z}^{n}$. Let $Q^{\prime}$ be the quotient of $G$ by this subgroup. Since $Q^{\prime}$ maps onto $Q$ with finite kernel, it is almost geometrically finite. Therefore we may assume that $V$ itself is free abelian of rank $n$. From Proposition 2.2 of [18], there exists a Seifert construction for this data, that is, a properly discontinuous action of $G$ on $\mathbf{R}^{n} \times L$, where $L$ is any contractible simplicial complex on which $Q$ acts properly discontinuously. The action takes $\mathbf{R}^{n}$-fibers (over points of $L$ ) to $\mathbf{R}^{n}$-fibers, and the quotient of each $\mathbf{R}^{n}$-fiber is a quotient of a compact flat manifold by a finite group action. Therefore $\left(\mathbf{R}^{n} \times L\right) / G$ is compact, so $G$ is almost geometrically finite.

\section{Laudenbach's Theorem.}

Throughout this section let $M$ be a Haken 3-manifold, $M \neq D^{3}$, and let $G \neq S^{2}$ be a compact connected properly imbedded incompressible surface in $M$. Let $m_{0}$ be a basepoint in $G$. If $G$ is not closed, choose $m_{0}$ in $\partial G$. Denote by $\operatorname{Imb}^{0}(G, M)$ the space of smooth imbeddings of $G$ in $M$ that take $m_{0}$ to $m_{0}$ and $\partial G$ to $\partial G$. The inclusion map of $G$ into $M$ is understood to be the basepoint of $\operatorname{Imb}^{0}(G, M)$. The following result is proven on pp. 49-62 of $[22]$.

Theorem 4.1. $\pi_{1}\left(\operatorname{Imb}^{0}(G, M)\right)=\{1\}$.

From this we deduce the result we will need.

Theorem 4.2. Assume that $G$ is not an annulus in a solid torus or a boundary-parallel disc. Let $f$ and $g$ be diffeomorphisms of $M$ which preserve $G$ and fix $m_{0}$. Let $H$ be an isotopy from $f$ to $g$, whose trace at $m_{0}$ lies in 
$\pi_{1}\left(G, m_{0}\right)$. Then $H$ is deformable relative to $M \times \partial I$ to an isotopy through diffeomorphisms that preserve G. Moreover,

(i) If $H$ is relative to $\partial G$, then the new isotopy may be chosen to be relative to $\partial G$.

(ii) If $H$ is relative to $\partial M$, then the new isotopy may be chosen to be relative to $\partial M$.

(iii) If $H$ is relative to $\partial M$, and $f$ and $g$ agree on $G$, then the new isotopy may be chosen to be relative to $G \cup \partial M$.

(iv) If $F$ is an incompressible surface (not necessarily connected) in $M$, disjoint from $G$, such that $H$ preserves $F$, then the new isotopy may be chosen to agree with $H$ on $F$.

Note that by virtue of part (iv), the theorem applies to $G$ that are not connected, provided that the trace condition is satisfied for a basepoint in each component of $G$.

Proof. Replacing $f$ by $g^{-1} f$, we may assume that $g$ is the identity. If $\partial G \neq \emptyset$ and the hypothesis of one of cases (i), (ii), or (iii) holds, then $H$ is already an isotopy relative to $m_{0}$. Otherwise, we may change $f$ by isotopy in a neighborhood of $G$ so that the trace of $H$ at $m_{0}$ is trivial, and then (using [24]) so that $H$ is an isotopy relative to $m_{0}$. Consequently $H$ induces the identity automorphism on $\pi_{1}\left(M, m_{0}\right)$.

Let $f_{1}$ be the restriction of $f$ to $G$. We will first reduce to the situation when $f_{1}$ is the identity. In case (iii), this already holds. In cases (i) and (ii), $H$ is an isotopy relative to $\partial G$ or $\partial M$. As in Lemma 7.3 of [34], $f_{1}$ is homotopic to the identity preserving $\partial G$, so $f_{1}$ is isotopic to the identity. Choose a basepoint in each component of $\partial G$. Suppose that $\gamma$ is an arc in $G$ connecting two of them. The restriction of $H$ to $\gamma$ gives an isotopy in $M$ from $f(\gamma)$ to $\gamma$, and since $\pi_{2}(M, G)$ is trivial (because $\pi_{2}(M)=0$ and $\pi_{1}(G) \rightarrow \pi_{1}(M)$ is injective), $f(\gamma)$ is homotopic and hence isotopic to $\gamma$ in $G$. Thus by changing $f$ by isotopy preserving $G$ and relative to $\partial M$, we may assume that $f_{1}$ preserves a set of arcs (disjoint except for their endpoints) connecting the basepoints in the boundary circles. This, together with the fact that $f_{1}$ is isotopic to the identity relative to $m_{0}$, implies that $f_{1}$ is isotopic to the identity relative to $\partial G$. After changing $f$ by isotopy preserving $G$ and relative to $\partial M$, we may assume that $f_{1}$ is the identity. Finally, suppose that none of (i), (ii), or (iii) holds. The trace condition implies that $f$ induces the identity automorphism on $\pi_{1}\left(M, m_{0}\right)$. Since $f(G)=G$, the restriction $f_{1}$ of $f$ to $G$ induces the identity automorphism on $\pi_{1}\left(G, m_{0}\right)$. Therefore $f_{1}$ is homotopic to the identity. Any orientationpreserving diffeomorphism of a compact 2-manifold that is homotopic to the identity is isotopic to the identity, unless $G$ is a disc or annulus and the diffeomorphism is orientation-reversing. If $f_{1}$ is orientation-reversing, then $f$ must reverse the sides of $G$. If $G$ were a disc, then since $f$ induces the 
identity automorphism on $\pi_{1}\left(M, m_{0}\right), M$ would have to be a 3-ball, so $G$ would be a boundary-parallel disc. If $G$ were an annulus, then for the same reason $M$ would have to be a solid torus, also excluded. So $f_{1}$ is isotopic to the identity, and since $f_{1}$ induces the identity automorphism on $\pi_{1}\left(G, m_{0}\right)$, we may assume that the isotopy preserves $m_{0}$. Extending such an isotopy of $f_{1}$ to an isotopy of $f$, we may assume that $f_{1}$ is the identity on $G$.

Next we need to achieve that $H$ preserves $\partial G$. In (i), (ii), or (iii), no alteration is needed. Otherwise, let $C$ be the component of $\partial G$ that contains $m_{0}$, let $B$ be the component of $\partial M$ that contains $C$, and consider the trace $\tau$ of $\left.H\right|_{B}$ at $m_{0}$. Note that $C$ is not contractible in $B$, since $G$ is not a boundary-parallel disc. Suppose that $\tau$ is not trivial in $\pi_{1}(B)$. Suppose for contradiction that $\tau$ does not lie in $\pi_{1}(C)$. Since $f$ is the identity on $C, \tau$ lies in the normalizer of $\pi_{1}(C)$ in $\pi_{1}(B)$. Therefore $B$ is a torus, and since $\tau$ is essential in $\pi_{1}(B)$ and trivial in $\pi_{1}(M), M$ must be a solid torus. Since $\tau$ is not in $\pi_{1}(C), C$ is not contractible in $\pi_{1}(M)$, so $G$ is an annulus, the case excluded by hypothesis. So we may assume that $\tau$ lies in $\pi_{1}(C)$. Since $\tau$ is trivial in $\pi_{1}(M), G$ must be a disc. There is an isotopy of $M$ that preserves $G$ and moves $m_{0}$ around $C$. Juxtaposing $H$ with the correct multiple of this isotopy, we may assume that $\tau$ is trivial in $\pi_{1}(B)$. By $[6], \pi_{1}\left(\operatorname{Imb}\left(\left(C, m_{0}\right),\left(B, m_{0}\right)\right)\right)$ is trivial unless $B$ is a 2 -sphere, a case excluded since $G$ is not a boundary-parallel disc, so $H$ may be deformed in a neighborhood of $B$ so that it preserves $C$ at each level. Repeating for the other components of $\partial G$, we may assume that $\partial G$ is preserved at each level of $H$.

The restriction $j_{t}$ of $H$ to $G \times I$ defines a loop in $\pi_{1}\left(\operatorname{Imb}^{0}(G, M)\right)$. Applying Theorem 4.1, this loop is contractible, so there exists a 2-parameter family $j_{t, s}, 0 \leq s, t \leq 1$, such that $j_{t, 0}=j_{t}$, and $j_{t, 1}, j_{0, s}$, and $j_{1, s}$ are the inclusions for each $t$ and $s$. Define $J_{t, 0}=H_{t}, J_{0, s}=f$, and $J_{1, s}=1_{M}$. By the isotopy extension theorem (i.e. the fact that $\operatorname{Diff}^{0}(M) \rightarrow \operatorname{Imb}^{0}(G, M)$ is a Serre fibration) this extends to a 2-parameter family $J_{t, s}$ of diffeomorphisms of $M$. Letting $K_{t}=J_{t, 1}$, we have an isotopy from $f$ to $1_{M}$ relative to $G$, and the existence of an isotopy preserving $G$ together with statements (i), (ii), and (iii) are established.

For (iv), notice that all of our alterations to $H$ may be performed so as not to change $H$ outside a neighborhood of $G$. Therefore if $F$ is another incompressible surface (not necessarily connected) which is preserved by $H$, we cut along $F$ and apply the previous argument to obtain a new isotopy agreeing with $H$ on $F$.

There is a 2-dimensional analogue of Theorem 4.2 .

Theorem 4.3. Let $G \neq S^{2}$ be a surface and $k$ an arc or circle essentially imbedded in $G$. If $k$ is a circle, let $m_{0}$ be a basepoint in $k$. Let $f$ and $g$ be diffeomorphisms of $G$ which preserve $G$. Let $H$ be an isotopy from $f$ to $g$ 
through diffeomorphisms fixing $m_{0}$, if $k$ is a circle, or preserving $\partial k$, if $k$ is an arc. Then $H$ is deformable relative to $G \times \partial I$ to an isotopy through diffeomorphisms that preserve $k$. Moreover,

(i) If $H$ is relative to $\partial M$, then the new isotopy may be chosen to be relative to $\partial M$.

(ii) If $H$ is relative to $\partial M$, and $f$ and $g$ agree on $G$, then the new isotopy may be chosen to be relative to $G \cup \partial M$.

(iii) If $\ell$ is a 1-manifold in $G$, disjoint from $k$, such that $H$ preserves $\ell$, then the new isotopy may be chosen to agree with $H$ on $\ell$.

The proof is analogous to the proof of Theorem 4.2, but much simpler.

\section{Exceptional Seifert-fibered 3-manifolds.}

In the next section we will give a general treatment of the mapping class groups of Seifert-fibered Haken 3-manifolds, but there are a few exceptional cases to which it will not apply. We address those cases in the present section. The manifolds (all assumed to be admissibly fibered with complete boundary pattern) are:

(E1) The $S^{1}$-bundle over the annulus, with boundary pattern $\underline{\underline{\emptyset}}$.

(E2) The $S^{1}$-bundle over the Möbius band, with boundary pattern $\underline{\underline{\emptyset}}$.

(E3) An $S^{1}$-bundle over the torus.

(E4) An $S^{1}$-bundle over the Klein bottle.

(E5) The Hantzsche-Wendt manifold, which is the closed flat 3-manifold with Seifert invariants $\left\{-1 ;\left(n_{2}, 1\right) ;(2,1),(2,1)\right\}$ (see [29] pp. 133, 138, [3] pp. 478-481, [7, 33, 36]).

(E6) A Haken manifold which fibers over $S^{2}$ with three exceptional orbits.

Proposition 5.1. Let $(\Sigma, \underline{\underline{\sigma}})$ be admissibly fibered as a Seifert 3-manifold of one of the exceptional types (E1)-(E4). Then $\mathcal{H}(\Sigma, \underline{\underline{\sigma}})$ is almost geometrically finite.

Proof. For (E1), from Proposition 3.4.1 of [26] we have $\mathcal{H}(\Sigma, \underline{\underline{\sigma}})$ isomorphic to $\mathbf{Z} / 2 \times \mathrm{GL}(2, \mathbf{Z})$ (the $\mathbf{Z} / 2$ factor is generated by reflection in the $I$-fibers of the $I$-bundle structure). So $\mathcal{H}(\Sigma, \underline{\underline{\sigma}})$ is virtually free. For $(\mathrm{E} 2), \mathcal{H}(\Sigma, \underline{\underline{\sigma}})$ is finite, by Proposition 3.4.2 of [26]. For (E3), if the Euler class is zero then $\Sigma$ is the 3 -torus, with $\mathcal{H}(\Sigma) \cong \operatorname{Out}\left(\pi_{1}(\Sigma)\right) \cong \mathrm{GL}(3, \mathbf{Z})$. If the Euler class is $n$, then $\pi_{1}(\Sigma) \cong\left\langle x, y, t \mid[x, t]=[y, t]=1,[x, y]=t^{n}\right\rangle$. The center is $\mathbf{Z}$ generated by $t$, and the quotient of $\pi_{1}(\Sigma)$ by its center is $\mathbf{Z} \times \mathbf{Z}$ generated by the images of $x$ and $y$. Sending $\operatorname{Out}\left(\pi_{1}(\Sigma)\right)$ to $\operatorname{Aut}(\mathbf{Z} \times \mathbf{Z})=\operatorname{GL}(2, \mathbf{Z})$ is surjective and splits (the three automorphisms determined by sending (1) $x$ to $x y, y$ to $y$, and $t$ to $t,(2) x$ to $x, y$ to $x y$, and $t$ to $t$, and (3) $x$ to $y$, $y$ to $x$, and $t$ to $t^{-1}$ define the splitting). Elements of the kernel are the automorphisms $\phi(i, j)$ that send $x$ to $x t^{i}, y$ to $y t^{j}$, and $t$ to $t$. Conjugation 
by $x$ equals $\phi(0, n)$ and conjugation by $y$ equals $\phi(-n, 0)$, so the kernel of $\operatorname{Out}\left(\pi_{1}(\Sigma)\right) \rightarrow \mathrm{GL}(2, \mathbf{Z})$ is $\mathbf{Z} / n \times \mathbf{Z} / n$, showing that $\mathcal{H}(\Sigma) \cong \operatorname{Out}\left(\pi_{1}(\Sigma)\right)$ is almost geometrically finite. The manifolds of type (E4) are analyzed in Proposition 3.4.4 of [26]. If the Euler class is zero then there is a homomorphism from $\operatorname{Out}\left(\pi_{1}(M)\right)$ to $\operatorname{PGL}(2, \mathbf{Z})$ with finite kernel. Since $\operatorname{PSL}(2, \mathbf{Z})$ is virtually free, it is almost geometrically finite. If the Euler class is nonzero, $\operatorname{Out}\left(\pi_{1}(\Sigma)\right)$ is finite.

Proposition 5.2. Let $M$ be one of the exceptional types (E5)-(E6). Then $\mathcal{H}(M)$ is finite.

Proof. For (E5), it is from [3] (although the correct structure of the group was later given in [36]). The lemma in Section 3.4 of [26] gives case (E6).

\section{Fibered 3-manifolds.}

We first treat the case of $I$-bundles, over surfaces which are not necessarily connected.

Lemma 6.1. Suppose that $(\Sigma, \underline{\underline{\sigma}})$ is admissibly I-fibered over the compact 2-manifold $(B, \underline{\underline{b}})$. Let $p: \Sigma \rightarrow B$ be the projection. Then $\mathcal{H}(\Sigma, \underline{\underline{\sigma}})$ is isomorphic to a semidirect product $F \circ \mathcal{H}(B, \underline{\underline{b}})$ where $F$ is a direct sum of copies of $\mathbf{Z} / 2$, one for each component of $\Sigma$, and the action of an element of $\mathcal{H}(B, \underline{\underline{b}})$ on $F$ is to permute the copies of $\mathbf{Z} / 2$ exactly as it permutes the corresponding components of $\Sigma$.

Proof. Let $i:(B, \underline{\underline{b}}) \rightarrow(\Sigma, \underline{\underline{\sigma}})$ be the 0 -section of the $I$-bundle (where $I$ is regarded as $[-1,1]$ and the structure group is reduced to $\mathbf{Z} / 2$ generated by reflection in $I$, and the twisting is given by the orientation homomorphism). For each component of $\Sigma$ there is the involution given by reflection in the $I$-fibers, and this is not admissibly isotopic to the identity since it is orientation-reversing. These give the generators of $F$.

Define $j: \mathcal{H}(B, \underline{\underline{b}}) \rightarrow \mathcal{H}(\Sigma, \underline{\underline{\sigma}})$ by extending diffeomorphisms on $i(B)$ to $\Sigma$ linearly in each fiber, choosing the unique way to do this that is orientationpreserving on $\Sigma$. To see that this is injective, let $\langle h\rangle$ be an element of $\mathcal{H}(B, \underline{\underline{b}})$ such that $j(\langle h\rangle)$ is trivial in $\mathcal{H}(\Sigma, \underline{\underline{\sigma}})$. Then $h$ is orientation-preserving, since otherwise the restriction of $j(h)$ of the lid or lids of $\Sigma$ cannot be isotopic to the identity. Projecting an admissible isotopy from $j(h)$ to $1_{\Sigma}$ down to $i(B)$ gives an admissible homotopy from $h$ to $1_{B}$. This implies that $h$ is admissibly isotopic to $1_{B}$ (see for example Lemma 2.19 of [2]). Corollary 5.9 of [17] shows that the image of $j$ is the entire group of orientation-preserving mapping elements of $\mathcal{H}(\Sigma, \underline{\underline{\sigma}})$. Then, it is clear that the subgroups $F$ and $j(\mathcal{H}(\Sigma, \underline{\underline{\sigma}}))$ generate $\mathcal{H}(\Sigma, \underline{\underline{\sigma}})$, and $F$ is normal, and the lemma follows.

Throughout the remainder of this section, $(\Sigma, \underline{\underline{\sigma}})$ will denote a Haken 3 -manifold with complete and useful boundary pattern, which admits an 
admissible Seifert fibering over $(B, \underline{b})$. A diffeomorphism of $\Sigma$ is called fiberpreserving if it carries each fiber $\Sigma$ to a fiber of $\Sigma$, and is called vertical if it takes each fiber to itself. By $\mathcal{H}_{f}(\Sigma, \underline{\underline{\sigma}})$ we indicate the mapping classes of fiber-preserving diffeomorphisms (that is, fiber-preserving diffeomorphisms modulo isotopy through fiber-preserving diffeomorphisms). There is a natural homomorphism $\mathcal{H}_{f}(\Sigma, \underline{\underline{\sigma}}) \rightarrow \mathcal{H}(\Sigma, \underline{\underline{\sigma}})$.

Theorem 6.2. If $(\Sigma, \underline{\underline{\sigma}})$ is not one of $(\mathrm{E} 1)-(\mathrm{E} 6)$, then $\mathcal{H}_{f}(\Sigma, \underline{\underline{\sigma}}) \rightarrow \mathcal{H}(\Sigma, \underline{\underline{\sigma}})$ is an isomorphism.

Proof. Provided that $(\Sigma, \underline{\underline{\sigma}})$ is not an exception (E1)-(E5), [33] or Theorem 8.1.7 of [29] shows that the homomorphism is surjective. If it is not an exception (E6), then it has a vertical incompressible surface, and the argument of p. 85-86 of [34] shows that it is injective.

Define $\mathcal{H}^{0}(\Sigma, \underline{\underline{\sigma}})$ to be the elements of $\mathcal{H}(\Sigma, \underline{\underline{\sigma}})$ that contain a vertical diffeomorphism. As in Lemma 25.2 of [17] (see also Lemma 3.5.3 of [26]), we have the following calculation.

Proposition 6.3. Let $\Sigma$ be Seifert-fibered over $(B, \underline{\underline{b}})$, with no component of $\Sigma$ an exceptional case (E1)-(E6). Then $\mathcal{H}_{+}^{0}(\Sigma, \underline{\underline{\sigma}}) \cong H_{1}(B,|\underline{\underline{b}}|)$.

The rough idea behind this result is that the generators of $\mathcal{H}_{+}^{0}(\Sigma, \underline{\underline{\sigma}})$ are Dehn twists (see [17] or Section 3.3 of [26] for a definition of Dehn twist) about vertical tori and annuli, which obey the same homological relations as their image circles and $\operatorname{arcs}$ in $B$. When $B$ is nonorientable, there is another type of generator supported in a neighborhood of a vertical Klein bottle; its square is a Dehn twist about the boundary of a regular neighborhood of the Klein bottle.

Let $E$ be the exceptional points of $B$, that is, the images of the exceptional orbits of $\Sigma$. This is a finite subset of the interior of $B$. Denote by $\rho: \mathcal{H}(\Sigma, \underline{\underline{\sigma}}) \rightarrow \mathcal{H}(B-E, \underline{\underline{b}})$ the homomorphism induced by projection of fiber-preserving homomorphisms to the base surface. Define $\mathcal{H}_{0}(B-E, \underline{\underline{b}})$ to be the subgroup of $\mathcal{H}(B-E, \underline{\underline{b}})$ consisting of the classes $\langle f\rangle$ such that $f$ is admissibly isotopic to a map which is the identity on $|\underline{b}|=\partial B$, and $f$ permutes the punctures of $B$ trivially. Since $\underline{\underline{b}}$ consists of arcs and circles (and since $B$ is of finite type), $\mathcal{H}_{0}(B-E, \underline{\underline{b}})$ has finite index in $\mathcal{H}(B-E, \underline{\underline{b}})$.

Proposition 6.4. The image of $\rho: \mathcal{H}_{f}(\Sigma, \underline{\underline{\sigma}}) \rightarrow \mathcal{H}(B-E, \underline{\underline{b}})$ contains $\mathcal{H}_{0}(B-E, \underline{\underline{b}})$, hence has finite index in $\mathcal{H}(B-E, \underline{\underline{b}})$. If $\partial B \neq \emptyset$, then there exists a homomorphism $s: \mathcal{H}_{0}(B-E, \underline{\underline{b}}) \rightarrow \mathcal{H}_{f}(\Sigma, \underline{\underline{\sigma}})$ such that $\rho s$ is the identity, and such that each $s(\langle f\rangle)$ has a representative which is the identity on $\partial \Sigma$.

Proof. The proposition follows from the special case when $B$ is connected. The connected case appears a Proposition 25.3 of [17] and Theorem 3.5.2 of [26]. The splitting is constructed using a cross-section $i: B-E \rightarrow \Sigma-\widetilde{E}$, 
where $\widetilde{E}$ is the union of the exceptional fibers. For $\langle f\rangle \in \mathcal{H}_{0}(B-E, \underline{\underline{b}})$, one may assume that $f$ is the identity on $\partial B$, so (since $s(f)$ is always selected to be orientation-preserving) that $s(f)$ is the identity on $i(\partial B)$ and hence on $\partial \Sigma$.

Propositions 6.3 and 6.4, combined with Lemma 3.2, yield immediately the following:

Theorem 6.5. Let $(\Sigma, \underline{\underline{\sigma}})$ be an admissibly Seifert-fibered Haken 3-manifold with complete and useful boundary pattern $\sigma$. Assume that $(\Sigma, \underline{\underline{\sigma}})$ is not one of the exceptional manifolds (E1)-(E6). Then there is an exact sequence

$$
1 \longrightarrow V \longrightarrow \mathcal{H}(\Sigma, \underline{\underline{\sigma}}) \longrightarrow Q \longrightarrow 1
$$

where $V$ has a finitely generated abelian subgroup of finite index, and $Q$ is almost geometrically finite.

Applying Proposition 3.3, we have:

Corollary 6.6. Let $(\Sigma, \underline{\underline{\sigma}})$ be an admissibly Seifert-fibered Haken 3-manifold with complete and useful boundary pattern $\sigma$. Assume that $(\Sigma, \underline{\underline{\sigma}})$ is not one of the exceptional manifolds (E1)-(E6). Then $\mathcal{H}(\Sigma, \underline{\underline{\sigma}})$ is almost geometrically finite.

\section{Haken manifolds.}

Throughout this section we assume that $(M, \underline{\underline{m}})$ is a Haken manifold with a complete and useful boundary pattern. We allow the possibility that $\partial M$ is empty. Denote by $\Sigma$ the characteristic submanifold of $(M, \underline{m})$. Let $\sigma$ be the proper boundary pattern on $\Sigma$. The following result was proved in [26], but we present a less abbreviated proof here. A reference for the Sol geometry is Theorem 5.5 of [31].

Proposition 7.1. Suppose that $M$ is not a torus bundle over the circle that admits a Sol structure. Then $\mathcal{H}(M, \Sigma, \underline{\underline{m}}) \rightarrow \mathcal{H}(M, \underline{\underline{m}})$ is an isomorphism.

Proof. Since the characteristic submanifold is unique up to admissible isotopy, the homomorphism is surjective. For injectivity, let $\langle f\rangle \in \mathcal{H}(M, \Sigma, \underline{\underline{m}})$ and suppose that $H: M \times I \rightarrow M$ is an admissible isotopy from $f$ to $1_{M}$. We must find an isotopy that preserves the frontier of $\Sigma$.

Let $F$ be a component of the frontier of $\Sigma$. We claim that $f(F)=F$. Suppose not. The restriction of $H$ to $F \times I$ is an admissible map from an $I$-bundle or Seifert fiber space into $(M, \underline{\underline{m}})$, so by Proposition 13.1 of [17], it is admissibly homotopic into $\Sigma$. That is, two components of the frontier of $\Sigma$ are admissibly homotopic in $\Sigma$. By Proposition 19.1 of [17], these components must be admissibly parallel in $\Sigma$, that is, the component of $\Sigma$ containing $F$ is of the form $F \times I$ and $f$ interchanges of the components of its boundary. Therefore $f$ is isotopic to a diffeomorphism that preserves $F$ 
and interchanges its sides. Since $f$ is isotopic to the identity, Lemma 7.4 of $[34]$ shows that this is impossible.

By isotopy preserving $\Sigma$, we may assume that $f$ fixes a basepoint $m_{0}$ in the interior of $F$. We claim that the trace of $H$ at $m_{0}$ is in the subgroup $\pi_{1}\left(F, m_{0}\right)$. When $\partial M$ is nonempty, Corollary 18.2 of $[\mathbf{1 7}]$ applies to prove the claim. When $\partial M$ is empty, the argument in Lemma 18.1 of [17] shows that if the claim is false then the components of $\Sigma$ and $\overline{M-\Sigma}$ adjacent to $F$ are each diffeomorphic to the product of the torus and an interval. By maximality of $\Sigma$, this is only possible when $M$ is a torus bundle over the circle which admits a Sol structure, which is excluded by hypothesis. This establishes the claim.

Since $F$ is a square, annulus, or torus, there is an isotopy on $F$ from the identity to the identity whose trace is equal to the trace of $H$. So it is possible to change $f$ by an admissible isotopy with support in a neighborhood of $F$, so that the trace of the isotopy from $f$ to the identity of $M$ is trivial. Then $f$ must induces the identity automorphism on $\pi_{1}\left(M, m_{0}\right)$. Since $f$ preserves $F$, it also induces the identity isomorphism on $\pi_{1}\left(F, m_{0}\right)$.

Let $f_{1}$ be the restriction of $f$ to $F$. We will show that $f_{1}$ preserves each element of the boundary pattern of $F$. If $F$ is a torus, there is nothing to prove. Suppose $F$ is an annulus, so that the boundary pattern consists of the two boundary circles. If $f_{1}$ interchanges them, then since $f_{1}$ induces the identity automorphism it must be orientation-reversing. But $f$ preserves the sides of $F$ (because $f$ preserves $\Sigma$, or alternatively using Lemma 7.4 of [34] again), so $f$ would be orientation-reversing and could not be isotopic to the identity. Suppose that $F$ is a square. Its boundary pattern consists of the four edges. Suppose for contradiction that $f_{1}$ moves some edge to a different edge. Since $f$ is admissibly homotopic to the identity, it must preserve each element of $\underline{\underline{m}}$. Since adjacent edges cannot lie in the same element of $\underline{\underline{m}}$, $f_{1}$ must interchange a pair of opposite edges. If it interchanges one pair of opposite edges, but preserves each of the other two edges, then $f_{1}$ is orientation-reversing, a contradiction as in the case when $F$ is an annulus. Therefore $f$ must interchange both pairs of opposite edges. Since $F$ is a square, the component of $\Sigma$ containing $F$ must be an $I$-bundle, and since opposite edges of $F$ lie in the same component of the boundary pattern, one pair lies in the lid and the other pair are joined by a square $S$ which is contained in an element $F^{\prime}$ of $\underline{m}$. Now the $I$-bundle cannot be fibered over the disc, because since $F$ and $S$ are sides which meet in two fibers, the $I$-bundle would be fibered over a 2 -faced disc and would not be essentially imbedded. This is impossible since it is a component of $\Sigma$. So the center circle of the annulus $F \cup S$ is essential in $M$. Since $f$ interchanges opposite edges of $F$, it must send this element of $\pi_{1}(M)$ to its negative, which is impossible since $f$ induces the identity automorphism on $\pi_{1}\left(M, m_{0}\right)$. 
Fix a component $F$ of the frontier of $\Sigma$. Suppose $F$ is a torus. Since the trace of the isotopy at $m_{0}$ is trivial, Theorem 4.2 applies to show that the isotopy from $f$ to the identity may be deformed to preserve $F$ at each stage.

Suppose $F$ is an annulus. Consider a basepoint $b_{0}$ in a boundary circle $C$ of $F$. Since $f_{1}$ is the identity on $F$, and the trace of $H$ at $m_{0}$ is trivial, the trace of $H$ at $b_{0}$ is also trivial in $\pi_{1}\left(M, b_{0}\right)$. Since the elements of $\underline{\underline{m}}$ are incompressible, the trace at $b_{0}$ is trivial in $\pi_{1}(G)$ where $G$ is the element of $\underline{\underline{m}}$ that contains $b_{0}$. Applying Theorem 4.3, we may deform the isotopy admissibly in a neighborhood of $\partial M$ so that $C$ preserved at each level of the isotopy. Repeating, we assume that all of $\partial F$ is preserved, and then apply Theorem 4.2.

Now suppose $F$ is a square. Let $b_{0}$ be a corner where two edges meet, so $b_{0} \in G_{1} \cap G_{2}$ for two elements of the boundary pattern. Again, the trace of $H$ at $b_{0}$ is trivial in $\pi_{1}\left(G_{1}\right)$ and $\pi_{1}\left(G_{2}\right)$. If it is not trivial in the fundamental group of the component $k$ of $G_{1} \cap G_{2}$ that contains it, then that component is a circle and $G_{1}$ and $G_{2}$ must be discs. Then $M$ would be a $3-$ ball with boundary pattern $\left\{G_{1}, G_{2}\right\}$ and $M$ could not contain the essentially imbedded square $F$. We conclude that the trace of $H$ at $b_{0}$ is trivial in $\pi_{1}(k)$, so we may assume that $H$ preserves $b_{0}$. Repeating, we assume that $H$ preserves each of the four corners of $F$. Now using Theorem 4.3 we may assume that $H$ preserves each of the four edges of $F$, and apply Theorem 4.2 to assume that $H$ preserves $F$.

Repeating this for each component of the frontier of $\Sigma$, not disturbing those already adjusted, shows that $f$ was the trivial element of $\mathcal{H}(M, \Sigma, \underline{\underline{m}})$.

From now until when we reach Theorem 7.7, we assume that $M \neq \Sigma$. Let $H=\overline{M-\Sigma}$, and let $\underline{\underline{h}}$ be the proper boundary pattern on $H$. Define $R_{H}$ to be the image of the restriction $\mathcal{H}(M, \Sigma, \underline{\underline{m}}) \rightarrow \mathcal{H}(H, \underline{\underline{h}})$. From Lemma 4.2.1 of $[\mathbf{2 6}]$ we have the following fact.

\section{Lemma 7.2. $R_{H}$ is finite.}

Actually, according to Theorem $2.1, \mathcal{H}\left(H_{i}, \underline{\underline{h_{i}}}\right)$ is itself finite for all components of $(H, \underline{\underline{h}})$ except the case when $\left(H_{i}, \underline{\underline{\underline{h_{i}}}}\right)=\left(T^{2} \times I, \underline{\underline{\emptyset}}\right)$. For these a special argument is given in [26]. The idea is that on the adjacent Seifertfibered component(s) of $\Sigma$, any diffeomorphism must be fiber-preserving up to isotopy. The fibers are linearly independent in $\pi_{1}\left(T^{2} \times I\right) \cong \mathbf{Z} \times \mathbf{Z}$, and only $\pm I$ can preserve two linearly independent elements of $\mathbf{Z} \times \mathbf{Z}$, allowing at most two possibilities for the restriction to $\mathcal{H}\left(H_{i}, \underline{\underline{h_{i}}}\right)$.

Since $M \neq \Sigma$, the components of $\Sigma$, with their proper boundary patterns, are of four kinds.

(i) The components which can be admissibly fibered as $I$-bundles with their lids in $\partial M$. Their union will be denoted by $I$. 
(ii) Those that are diffeomorphic to $S^{1} \times S^{1} \times I$ with boundary pattern $\bar{\phi}$, but are not as in (i) (that is, exceptional case (E1)). These must $\overline{\mathrm{b}}$ e Seifert-fibered (since as $I$-bundles they could not have both lids in $\partial M)$, and must have frontier equal to one or both of their boundary components. Their union will be denoted by $T$.

(iii) Those that are diffeomorphic to the twisted $I$-bundle over the Klein bottle with boundary pattern $\bar{\phi}$ (that is, exceptional case (E2)). Their union will be denoted by $K$.

(iv) The components that are not as in (i), (ii), or (iii). These are Seifertfibered, since they are not as in (i), and their fiberings are unique up to admissible isotopy, since they are not as in (ii) or (iii). Their union will be denoted by $S$.

The proper boundary pattern of $I$ is denoted $\underline{\underline{i}}$, and similarly for $K, T$, and $S$. Clearly each of $I, K, T$, and $S$ is preserved by each element of $\mathcal{H}(M, \Sigma, \underline{\underline{m}})$. Let $R_{T}$ be the image of the restriction $\mathcal{H}(M, \Sigma, \underline{\underline{m}}) \rightarrow \mathcal{H}(T, \underline{\underline{t}})$.

Lemma 7.3. $R_{T}$ is finite.

Proof. Consider the composition

$$
\mathcal{H}(M, \Sigma, \underline{\underline{m}}) \rightarrow R_{T} \rightarrow \mathcal{H}(\operatorname{Fr}(T)),
$$

where $\operatorname{Fr}(T)$ is the frontier. Since this equals the composition

$$
\mathcal{H}(M, \Sigma, \underline{\underline{m}}) \rightarrow \mathcal{H}(H, \underline{\underline{s}}) \rightarrow \mathcal{H}(\operatorname{Fr}(T)),
$$

and $\mathcal{H}(M, \Sigma, \underline{\underline{m}}) \rightarrow \mathcal{H}(H, \underline{\underline{s}})$ has finite image by Lemma 7.2 , it follows that $R_{T} \rightarrow \mathcal{H}(\operatorname{Fr}(\bar{T}))$ has finite image. But it is also injective, since any diffeomorphism of $T$ isotopic to the identity on a boundary component is isotopic to the identity. Therefore $R_{T}$ is finite.

Lemma 7.4. $\mathcal{H}(K, \underline{\underline{k}})$ is finite.

Proof. Since $(K, \underline{\underline{k}})$ fibers admissibly as an $I$-bundle, $\underline{\underline{k}}=\underline{\underline{\emptyset}}$. By Lemma 6.1, if $X$ is the twisted $I$-bundle over the Klein bottle $Y$, we have $\mathcal{H}(X, \underline{\underline{\emptyset}}) \cong \mathcal{H}(Y)$, and by [23] the latter is $\mathbf{Z} / 2 \times \mathbf{Z} / 2$. Since $K$ has finitely many components, $\mathcal{H}(K, \underline{\underline{k}})$ is also finite.

Consider the restriction homomorphism

$$
\mathcal{H}(M, \Sigma, \underline{\underline{m}}) \rightarrow R_{H} \times R_{T} \times \mathcal{H}(K, \underline{\underline{k}}) \times \mathcal{H}(I, \underline{\underline{i}}) \times \mathcal{H}(S, \underline{\underline{s}}) .
$$

Let $(B, \underline{\underline{b}})$ be the quotient surface of $(S, \underline{\underline{s}})$, and let $E$ be the image of the exceptional fibers. By Theorem 6.2 and Proposition 6.4, there is a homomorphism $\mathcal{H}(S, \underline{\underline{s}}) \rightarrow \mathcal{H}(B-E, \underline{b})$, and composing with this in the $\mathcal{H}(S, \underline{\underline{s}})$ factor, we obtain a homomorphism

$$
\Psi: \mathcal{H}(M, \Sigma, \underline{\underline{m}}) \rightarrow R_{H} \times R_{T} \times \mathcal{H}(K, \underline{\underline{k}}) \times \mathcal{H}(I, \underline{\underline{i}}) \times \mathcal{H}(B-E, \underline{\underline{b}}) .
$$

Proposition 7.5. The image of $\Psi$ has finite index. 
Proof. By Lemmas 7.2, 7.3, and 7.4, we need only examine $\mathcal{H}(I, \underline{\underline{i}})$ and $\mathcal{H}(B-E, \underline{b})$. Let $\mathcal{H}_{0}(I, \underline{\underline{i}})$ be the subgroup of $\mathcal{H}(I, \underline{\underline{i}})$ consisting of the elements containing representatives which are the identity on the frontier of $I$, and recall the subgroup $\mathcal{H}_{0}(B-E, \underline{b})$ of $\mathcal{H}(B-E, \underline{b})$ defined shortly before Proposition 6.4. Since the mapping class groups of the square, annulus, arc, and circle are finite, these are finite index subgroups. It suffices to show that $\mathcal{H}_{0}(I, \underline{i}) \times \mathcal{H}_{0}(B-E, \underline{b})$ is contained in the image of $\Psi$.

Proposition 6.4 shows that the image of the homomorphism $\rho: \mathcal{H}_{f}(S, \underline{\underline{s}}) \rightarrow$ $\mathcal{H}(B-E, \underline{\underline{b}})$ contains $\mathcal{H}_{0}(B-E, \underline{\underline{b}})$, and that there is a homomorphism $s: \mathcal{H}_{0}(B-E, \underline{\underline{b}}) \rightarrow \mathcal{H}_{f}(S, \underline{\underline{s}})$ with $\rho s$ equal to the identity. Moreover, each $s(\langle h\rangle)$ can be represented by a diffeomorphism which is the identity on $\partial S$. So given an element in $\mathcal{H}_{0}(I, \underline{\underline{i}}) \times \mathcal{H}_{0}(B-E, \underline{\underline{b}})$, it can be represented on $I \cup S$ by a diffeomorphism which is the identity on the frontier of $I \cup S$. Extending this to $M$ using the identity map on $M-(I \cup S)$ produces an element of $\mathcal{H}(M, \Sigma, \underline{\underline{m}})$ that $\Psi$ carries to the given element.

Define $\mathcal{K}(M, \Sigma, \underline{\underline{m}})$ to be the kernel of $\Psi$.

Proposition 7.6. $\mathcal{K}(M, \Sigma, \underline{\underline{m}})$ is finitely generated and abelian.

Proof. Let $g$ be a vertical diffeomorphism of $S$ which is isotopic to the identity on $\operatorname{Fr}(S)$. Each such $g$ extends to a diffeomorphism of $M$ which is the identity outside a regular neighborhood of $S$. The image of $\mathcal{K}(M, \Sigma, \underline{\underline{m}})$ in $\mathcal{H}(S, \underline{\underline{s}})$ is contained in the subgroup of $\mathcal{H}_{+}^{0}(S, \underline{\underline{s}})$ consisting of elements representable by maps which are isotopic to the identity on the frontier of $S$. By Proposition $6.3, \mathcal{H}_{+}^{0}(S, \underline{\underline{s}})$ is finitely generated and abelian, hence so is the image of $\mathcal{K}(M, \Sigma, \underline{\underline{m}})$. Choose a set of generators for the image, and for each an extension to $M$. Let $X_{1}$ be the resulting subset of $\mathcal{H}(M, \Sigma, \underline{\underline{m}})$. These commute with each other and with any diffeomorphism supported in a regular neighborhood of $\operatorname{Fr}(H)$. Choose a set of generators $X_{2}$ (Dehn twists about components of $\operatorname{Fr}(H))$ for the elements of $\mathcal{H}(M, \Sigma, \underline{\underline{m}})$ representable by diffeomorphisms supported in a regular neighborhood of $\operatorname{Fr}(H)$. The set $X=X_{1} \cup X_{2}$ is a finite set of commuting elements, and we claim that it generates $\mathcal{K}(M, \Sigma, \underline{\underline{m}})$. Given any element of $\mathcal{K}(M, \Sigma, \underline{\underline{m}})$, we may compose this element with elements in $X_{1}$ to assume that its restriction to $S$ is isotopic to the identity. But all elements of $\mathcal{K}(M, \Sigma, \underline{\underline{m}})$ are isotopic to the identity when restricted to each of $I, K, T$, and $H$, so when the restriction to $S$ is also isotopic to the identity, the element is a product of elements in $X_{2}$.

Theorem 7.7. Let $M$ be a Haken manifold and $\underline{\underline{m}}$ a boundary pattern on $M$ whose completion is useful. Then $\mathcal{H}(M, \underline{\underline{m}})$ is virtually torsionfree and almost geometrically finite. 
Proof. If $M$ is a torus bundle over $S^{1}$ that admits a $S o l$ structure, then by Proposition 4.1 .2 of $[\mathbf{2 6}], \mathcal{H}(M)$ is finite. Otherwise, by Proposition 7.1, we may work instead with $\mathcal{H}(M, \Sigma, \underline{\underline{m}})$.

First we combine previous results to prove that $\mathcal{H}(M, \underline{m})$ is almost geometrically finite. If $M=\Sigma$, then Lemma 6.1 and Corollary 6.6 apply. Suppose that $M \neq \Sigma$. By Lemma $6.1, \mathcal{H}(I, \underline{\underline{i}})$ is almost geometrically finite. By Proposition 7.5, the image of $\Psi$ is almost geometrically finite. By Proposition 7.6, the kernel of $\Psi$ is finitely generated abelian. By Proposition 3.3, $\mathcal{H}(M, \underline{\underline{m}})$ is almost geometrically finite.

It was proven in $[\mathbf{2 6}]$ that $\mathcal{H}(M, \underline{\underline{m}})$ is virtually torsionfree, but we give here a proof that is much simpler.

If $M=\Sigma$, then Lemma 3.5.9 of [26] completes the proof, so we assume that $M \neq \Sigma$. Let $(Q, \underline{q})$ be obtained as follows. Let $\left(B_{1}, \underline{\underline{b_{1}}}\right)$ be the base surface of $(I, \underline{\underline{i}})$, and let $\left(\overline{\bar{B}}_{2}, \underline{\underline{b_{2}}}\right)$ be the base surface of $(S, \underline{\underline{s}})$, and $E$ the image of the exceptional fibers. Remove each component $(X, \underline{\underline{x}})$ from $\left(B_{1}, \underline{b_{1}}\right) \cup\left(B_{2}, \underline{b_{2}}\right)$ for which $\mathcal{H}(X-E, \underline{\underline{x}})$ is finite, and call the remainder $(Q, \underline{\underline{q}})$. Let

$$
\pi: R_{H} \times R_{T} \times \mathcal{H}(K, \underline{\underline{k}}) \times \mathcal{H}(I, \underline{\underline{i}}) \times \mathcal{H}\left(B_{2}-E, \underline{\underline{b_{2}}}\right) \longrightarrow \mathcal{H}(Q-E, \underline{\underline{q}})
$$

be the projection, and note that $\pi$ has finite kernel. Put $\Psi^{\prime}=\pi \Psi$ and $V=\left(\Psi^{\prime}\right)^{-1}(F)$ where $F$ is the subgroup of $\mathcal{H}(I, \underline{\underline{i}})$ from Lemma 6.1. Then we have an exact sequence

$$
1 \rightarrow V \longrightarrow \mathcal{H}(M, \underline{\underline{m}}) \stackrel{\Psi^{\prime}}{\longrightarrow} \mathcal{H}(Q-E, \underline{\underline{q}})
$$

where $V$ is virtually abelian. Let $\mathcal{G}(Q-E, \underline{q})$ denote the subgroup of $\mathcal{H}(Q-E, \underline{q})$ consisting of the elements that preserve each element of $\underline{\underline{q}}$ and each point of intersection of two elements of $\underline{\underline{q}}$, and let $\mathcal{G}_{0}(Q-E, \underline{\underline{q}})$ be the corresponding subgroup of $\mathcal{H}_{0}(Q-E, \underline{q})$. Let $\mathcal{H}^{\prime}(M, \underline{\underline{m}})$ denote the subgroup $\left(\Psi^{\prime}\right)^{-1}\left(\mathcal{G}_{0}(Q-E, \underline{q})\right)$; as in Proposition 6.4 , there is a splitting $s: \mathcal{G}_{0}(Q-E, \underline{\underline{q}}) \rightarrow \mathcal{H}^{\prime}(M, \underline{\underline{m}})$ so we have a semidirect product

$$
\mathcal{H}^{\prime}(M, \underline{\underline{m}})=V \circ \mathcal{G}_{0}(Q-E, \underline{\underline{q}}) .
$$

Let $W=H_{1}\left(B_{1}\right) \times V$, and consider the holomorph of $W, W \circ \operatorname{Aut}(W)$, which is defined to be the semidirect product in which $\operatorname{Aut}(W)$ acts naturally on $W$. We claim that $\operatorname{Aut}(W)$ is virtually torsionfree. Write $W$ as $W_{1} \circ T$ where $W_{1}$ is free abelian and $T$ is finitely generated. Since $T$ is characteristic, there is a homomorphism $\operatorname{Aut}(W) \rightarrow \operatorname{Aut}\left(W_{1}\right)$. It is easily checked that the kernel is a finite group of the form $\operatorname{Hom}\left(W_{1}, T\right) \circ \operatorname{Aut}(T)$, and moreover it splits. Since $\operatorname{Aut}\left(W_{1}\right) \cong \mathrm{GL}(n, \mathbf{Z})$ is virtually torsionfree, it follows that $\operatorname{Aut}(W)$ is virtually torsionfree. Since $W$ is also virtually torsionfree, and $W$ is finitely generated, Lemma 6.8 of $[\mathbf{2 8}]$ implies that $W \circ \operatorname{Aut}(W)$ is virtually torsionfree. 
The action in the semidirect product $V \circ \mathcal{G}_{0}(Q-E, \underline{\underline{q}})$ together with the natural action of $\mathcal{H}_{0}\left(B_{1}\right)$ on $H_{1}\left(B_{1}\right)$ defines a homomorphism from $\mathcal{G}_{0}(Q-$ $E, \underline{q})$ to $W \circ \operatorname{Aut}(W)$. Since the latter is virtually torsionfree, it suffices to show that the kernel is torsionfree. The kernel is contained in the subgroup of $\mathcal{G}_{0}(Q-E, \underline{q})$ consisting of elements that act trivially on $H_{1}(Q-E)$. Since we have removed all disc components from $Q-E$, elements of the kernel preserve each component of $Q-E$, so we may assume that $Q-E$ is connected. Let $P$ consist of one point from each arc of $\underline{q}$ together with all points that are intersections of two distinct $\operatorname{arcs}$ of $\underline{q}$. It is easy to see that $\mathcal{G}_{0}(Q-E, \underline{q})$ is isomorphic to a subgroup of finite index in $\mathcal{H}(Q-E \operatorname{rel} P)$. If $P$ is empty, then it is well-known that the subgroup of $\mathcal{H}(Q-E)$ inducing the identity on homology is torsionfree (see for example [4]). Suppose $P$ is not empty. Let $\operatorname{Diff}_{1}(Q-E)$ denote the subgroup of $\operatorname{Diff}(Q-E)$ consisting of the elements which induce the identity on $H_{1}(Q-E)$ and preserve each component of $\partial(Q-E)$. There is a fibration

$$
\operatorname{Diff}_{1}(Q-E \text { rel } P) \rightarrow \operatorname{Diff}_{1}(Q-E) \rightarrow \operatorname{imb}(P, \partial(Q-E))
$$

where $\operatorname{imb}(P, \partial(Q-E))$ is the connected component of the inclusion in the space of imbeddings $\operatorname{Imb}(P, \partial(Q-E))$. Clearly $\operatorname{imb}(P, \partial(Q-E)) \cong\left(S^{1}\right)^{k}$, where $k$ is the number of boundary circles of $Q-E$ that contain points of $P$. From the homotopy exact sequence of this fibration, we have an exact sequence

$$
0 \rightarrow \pi_{1}\left(\operatorname{Diff}_{1}(Q-E)\right) \rightarrow \mathbf{Z}^{k} \rightarrow \mathcal{H}_{1}(Q-E \text { rel } P) \rightarrow \mathcal{H}_{1}(Q-E) \rightarrow 1
$$

where $\mathcal{H}_{1}(Q-E$ rel $P)=\pi_{0}\left(\operatorname{Diff}_{1}(Q-E\right.$ rel $\left.P)\right)$ and $\mathcal{H}_{1}(Q-E)=$ $\pi_{0}\left(\operatorname{Diff}_{1}(Q-E)\right)$. Now $\pi_{1}\left(\operatorname{Diff}_{1}(Q-E)\right)$ is nontrivial only when $Q-E$ is an annulus and $k=2$ (a disc, a Möbius band, or an annulus with $k=1$ have already been excluded by the definition of $Q-E)$, and in this case the generator of $\pi_{1}\left(\operatorname{Diff}_{1}(Q-E)\right)$ is carried to an element of the form $( \pm 1, \pm 1) \in \mathbf{Z} \times \mathbf{Z}$. Therefore we have an exact sequence

$$
0 \rightarrow \mathbf{Z}^{\ell} \rightarrow \mathcal{H}_{1}(Q-E \text { rel } P) \rightarrow \mathcal{H}_{1}(Q-E) \rightarrow 1 .
$$

We have already seen that $\mathcal{H}_{1}(Q-E)$ is torsionfree, and therefore $\mathcal{H}_{1}(Q-$ $E$ rel $P$ ) is torsionfree.

To deduce some corollaries, it is convenient to introduce a general technique for extending results about $\mathcal{H}(M, \underline{\underline{m}})$ to relative mapping class groups. Assume that $\underline{\underline{m}}$ is complete. Suppose $\underline{\underline{m_{1}}} \subset \underline{\underline{m}}$. Take a fine triangulation of $\left|\underline{m_{1}}\right|$, which includes as vertices the points of $\partial\left|m_{1}\right|$ that are intersections of three elements of $\underline{\underline{m}}$, and let $\underline{\underline{m_{2}}}$ be the complete boundary pattern on $M$ consisting of the elements of $\underline{\underline{\underline{m}}}-\underline{m_{1}}$ and the 2-cells dual to the triangulation of $\left|\underline{\underline{m_{1}}}\right|$. Choose the triangulation so that at every vertex in $\left|\underline{\underline{m_{1}}}\right|$, at least four triangles meet (for example, take the barycentric subdivision of 
the triangulation of $\left|m_{1}\right|$ without introducing the barycenters of 1-simplices that lie in the boundary). Then, each dual 2-cell has at least four sides. This ensures the following.

(i) If $\underline{\underline{m}}$ is useful, then $\underline{\underline{m_{2}}}$ is useful.

(ii) If $\underline{\underline{m}}-\underline{\underline{m_{1}}}$ consists of the components of $\overline{\partial M-\left|\underline{\underline{m_{1}}}\right|}$, and these components are incompressible, then $\underline{\underline{m_{2}}}$ is useful.

The important property of $\underline{\underline{m_{2}}}$ is the following.

Proposition 7.8. $\mathcal{H}\left(M, \underline{\underline{m}}\right.$ rel $\left.\left|\underline{\underline{m_{1}}}\right|\right)$ is isomorphic to a subgroup of finite index in $\mathcal{H}\left(M, \underline{\underline{m_{2}}}\right)$.

Proof. Consider the natural homomorphism from $\mathcal{H}\left(M, \underline{\underline{m}}\right.$ rel $\left.\left|\underline{\underline{m_{1}}}\right|\right)$ to $\mathcal{H}\left(M, \underline{\underline{m_{2}}}\right)$. We claim this is injective and has image of finite index. Suppose

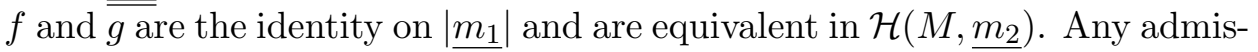
sible isotopy must preserve the intersection of any collection of dual 2-cells, so must preserve the points where three dual 2-cells in $W$ meet. Therefore we may assume the isotopy is relative to the dual 0-cells. Also, it must preserve the intersection of any two dual 2-cells, i.e. the dual 1-cells. By the Alexander trick applied to each dual 1 -cell $\times I$, we may make the admissible isotopy to be relative to the intersections of the dual cells. Then, by the Alexander trick applied to each dual 2-cell $\times I$, we may make the isotopy relative to all of $\left|\underline{m_{1}}\right|$. To show the image has finite index, let $\mathcal{H}\left(M, \underline{m_{2}}\right)$ act on the union of the set of all the dual cells. This defines a homomorphism to a finite permutation group, and by an argument similar to the proof of injectivity one shows that any element in the kernel is admissibly (for $\underline{\underline{m_{2}}}$ ) isotopic to the identity on $\left|\underline{\underline{m_{1}}}\right|$, so is in the image.

Using $\underline{\underline{m_{2}}}$ we can now deduce the corollaries of the main theorem.

Corollary 7.9. Let $(M, \underline{\underline{m}})$ be Haken with complete and useful boundary pattern, and let $W$ be a union of elements of $\underline{\underline{m}}$. Then $\mathcal{H}(M, \underline{\underline{m}}$ rel $W)$ is virtually torsionfree and almost geometrically finite.

Proof. By remark (i) above and Proposition 7.8, there is a complete and useful boundary pattern $m_{2}$ on $M$ so that $\mathcal{H}(M, \underline{\underline{m}}$ rel $W)$ is isomorphic to a subgroup of finite index in $\mathcal{H}\left(M, \underline{\underline{m_{2}}}\right)$. By Theorem $7.7, \mathcal{H}\left(M, \underline{\underline{m_{2}}}\right)$ is almost geometrically finite, hence so is $\overline{\overline{\mathcal{H}}}(M, \underline{\underline{m}}$ rel $W)$.

Corollary 7.10. Let $M$ be a Haken 3-manifold and let $W$ be a compact 2dimensional submanifold of $\partial M$ such that $\partial M-W$ is incompressible. Then $\mathcal{H}(M \mathrm{rel} W)$ is virtually torsionfree and almost geometrically finite.

Proof. Let $\underline{\underline{m_{1}}}$ be the set of components of $W$, and let $\underline{\underline{m}}$ be the union of $\underline{\underline{m_{1}}}$ with the set of components of $\overline{\partial M-W}$. The boundary pattern constucted above $\frac{m_{2}}{m_{2}}$ is complete, and by (ii) above it is useful. Since $\mathcal{H}(M$ rel $W)=$ $\mathcal{H}\left(M, \underline{\underline{\underline{m_{2}}}}\right.$ rel $\left.\left|\underline{\underline{m_{1}}}\right|\right)$, Corollary 7.9 applies. 


\section{The Kontsevich Conjecture.}

Throughout this section, the symbol $f \cong g$ means that $f$ and $g$ are isotopic relative to the boundary of the manifold on which they are defined. We first isolate a couple of technical steps that will be needed in later arguments.

Lemma 8.1. Let $M$ be a Haken manifold containing an incompressible surface $G$, not necessarily connected. Let $f$ and $g$ be two diffeomorphisms of $M$ which are homotopic relative to $\partial M$. Then $f \cong g$. If $f$ and $g$ agree on $G$, and the homotopy has trivial trace at a basepoint in each component of $G$, then they are isotopic relative to $G \cup \partial M$.

Proof. Replacing $f$ by $g^{-1} f$, we may assume that $f$ is orientation-preserving, $g$ is the identity, and $f$ restricts to the identity on $G$. As in Theorem II.6.1 of [22], the homotopy can be deformed relative to $M \times \partial I \cup \partial M \times I$ to an isotopy. In particular, the traces at basepoints on components of $G$ remain trivial. By Theorem 4.2, we may assume that the isotopy is relative to $G \cup \partial M$.

Lemma 8.2. Let $M$ be a compact 3-manifold, each of whose components is Haken with incompressible boundary. Assume that each component $M_{0}$ of $M$ has the property that if $g$ is a diffeomorphism from $M_{0}$ to itself such that $g^{n} \cong 1_{M_{0}}$, then there exists a diffeomorphism $h$ of $M_{0}$ such that $g \cong h$ with $h^{n}=1_{M_{0}}$. Then $M$ itself has this property.

Proof. It suffices to consider the case when $g$ acts transitively on the components of $M$. Let $M_{1}$ be a component and let $i$ be the smallest positive integer such that $g^{i}$ preserves $M_{1}$. Since $M_{1}$ has the property, $g^{i} \cong h$ such that $h^{n / i}=1_{M_{1}}$. Let $M_{2}=g^{-1}\left(M_{1}\right)$. By isotopy relative to the boundary of $M_{2}$, we may change $\left.g\right|_{M_{2}}$ to $h g^{1-i}$, then $g$ has order $n$ on each component of $M$.

We will need an extension of Theorem 3 of [15].

Proposition 8.3. Let $M$ be Haken with nonempty incompressible boundary. Assume that each component of $\partial M$ is a torus. If $g$ is a diffeomorphism from $M$ to itself such that $g^{n} \cong 1_{M}$, then $g \cong h$ such that $h^{n}=1_{M}$.

Proof. Consider the characteristic submanifold $\Sigma$ of $(M, \underline{\underline{\emptyset}})$. Since $\partial M$ consists of tori, each component of the frontier of $\Sigma$ is a torus, and is boundary parallel exactly when it lies in a component of $\Sigma$ which is a regular neighorhood of a component of $\partial M$. We induct on the number of components of the frontier of $\Sigma$ that are not parallel onto $\partial M$. If there are none, $M$ is either simple or a Seifert fiber space. By Theorem 3 of [15] and Lemma 8.1, the theorem is true for $M$. (In the remainder of the proof, Lemma 8.1 must be used in similar fashion to strengthen conclusions from [15], but we will no longer mention these individually.) 
We induct on the number of components of the frontier of $\Sigma$ that are not parallel into $\partial M$. Since $\Sigma$ is unique up to isotopy, we may assume that $g(\Sigma)=\Sigma$. Let $F$ be a component of the frontier of $\Sigma$ such that $F$ is not parallel into $\partial M$. Let $\widehat{F}=\cup g^{i}(F)$, a collection of components of the frontier of $\Sigma$. By induction and Lemma 8.2, it suffices to show that $g^{n}$ is isotopic to $1_{M}$ relative to $F \cup \partial M$.

Fix an isotopy $H: g^{n} \cong 1_{M}$. The proof of Lemma 9(ii) of [15] contains most of the arguments needed to obtain our conclusion, so we only explain the changes needed. We refer to the notation used there. The first paragraph of the proof (of Case (ii)) in [15] is not needed; since $\pi_{1}(M)$ is centerless the condition $h(\gamma) \simeq \gamma$ holds automatically, as shown by the argument for Lemma 6 of [15]. For the next paragraph, we know that $M$ cannot fiber over $S^{1}$ with fiber $F$, since $\partial M$ is nonempty, therefore the first half of the paragraph shows that the restriction of the homotopy to $\widehat{F} \times I$ is homotopic relative to $\widehat{F} \times \partial I$ to a map into $\widehat{F} \times I$. Therefore the homotopy at the start of the third paragraph may also be assume to be relative to $\partial M$. The remainder of the proof makes some rather delicate adjustments to achieve that the restriction of $g$ to $\widehat{F}$ is periodic and that the homotopy has trivial trace at a basepoint in each component of $\widehat{F}$. Since these changes take place only in a regular neighborhood of $\widehat{F}$, the resulting homotopy is still relative to $\partial M$. Then Lemma 8.1 yields from this an isotopy relative to $F \cup \partial M$, to complete the inductive step and the proof.

Theorem 8.4. Let $M$ be a Haken 3-manifold such that $\partial M$ is nonempty and incompressible. Then $\mathcal{H}(M$ rel $\partial M)$ is torsionfree.

Proof. Let $\langle g\rangle \in \mathcal{H}(M$ rel $\partial M)$ and suppose that for $n>1, g^{n} \cong 1_{M}$. We must show that $g \cong 1_{M}$.

Let $T$ be the union of the torus boundary components of $M$. Suppose first that $T=\partial M$. By Proposition 8.3, $g \cong h$ with $h^{n}=1_{M}$. Since $h$ is the identity on $\partial M$, a theorem of M. H. A. Newman (see Proposition 3.1 of [21]) shows that $h=1_{M}$. Now suppose that $T$ is not empty but $T \neq \partial M$. Form $N$ by gluing two copies of $M$ together along $\partial M-T$, and let $D(g)$ be the diffeomorphism of $N$ defined by taking $g$ on each copy of $M$. Since $D(g)^{n} \cong 1_{N}$, the previous case shows that $D(g) \cong 1_{N}$. Let $H: N \times I \rightarrow N$ be an isotopy from $D(g)$ to $1_{N}$. By Lemmas 7.2 and 7.3 of [34], $H$ may be deformed to a homotopy that preserves $G$. Therefore the trace of $H$ at a point in $G$ lies in $G$. Since $D(g)$ is the identity on $G$, the trace is a central element of $\pi_{1}(G)$. Since $G$ is not a torus, the center of $\pi_{1}(G)$ is trivial. By Theorem 4.2, $H$ may be deformed to an isotopy relative to $G$. Repeating, we have an isotopy relative to $\partial M-W$, so $g \cong 1_{M}$. This completes the case when $T$ is not empty.

Now suppose that no component of $\partial M$ is a torus. Let $G$ be a boundary component, and choose an essential simple closed curve $\gamma$ in $G$. Let $G_{1}$ be 
a regular neighborhood of $\gamma$ in $G$. Let $W$ be $S^{1} \times S^{1} \times I$, and let $G_{2}$ be a regular neighborhood of $S^{1} \times\left\{s_{0}\right\} \times\{0\}$ in $S^{1} \times S^{1} \times\{0\}$ for some $s_{0} \in S^{1}$. Form $N$ by identifying $G_{1}$ with $G_{2}$ and let $G_{0}$ be the incompressible surface in $N$ obtained from $G_{1}$ and $G_{2}$. Since $G_{0}$ is incompressible in $M$ and $W$, $N$ is Haken. Extend $g$ to a diffeomorphism $f$ of $N$ using the identity on $W$. The isotopy $g^{n} \cong 1_{M}$ extends using the identity on $W$ to an isotopy $f^{n} \cong 1_{N}$. Since $N$ has a torus boundary component, the previous case implies that $f \cong 1_{N}$. By Theorem $4.2, f \cong 1_{N}$ relative to $G_{1}$, and therefore $g \cong 1_{M}$.

Now we will weaken the hypothesis.

Theorem 8.5. Let $M$ be a Haken 3-manifold and let $F$ be a nonempty compact 2-manifold in $\partial M$. Then $\mathcal{H}(M$ rel $F)$ is torsionfree.

Proof. Fix $\langle g\rangle \in \mathcal{H}(M$ rel $F)$ with $g^{n}$ isotopic to $1_{M}$ relative to $F$ for some $n>1$. We must prove that $g$ is isotopic to $1_{M}$ relative to $F$.

Let $W$ be the union of the boundary components of $M$ that meet $F$. On each component $X$ of $\overline{W-F}$, we have $\left.g^{n}\right|_{X} \cong 1_{X}$. Since $\partial X$ is nonempty, Lemma 1.2 of $[\mathbf{1 4}]$ shows that $\mathcal{H}(X$ rel $\partial X)$ is torsionfree. Therefore $\left.g\right|_{X} \cong$ $1_{X}$, so we may change $g$ by isotopy relative to $F$ so that $\left.g\right|_{W}=1_{W}$. Also, $\pi_{1}(\operatorname{Diff}(X$ rel $\partial X))$ is trivial, so we may assume that $g^{n}$ is isotopic to $1_{M}$ relative to $W$.

Form $N$ by attaching two copies of $M$ along $\partial M-W$. Then $D(g)^{n} \cong 1_{N}$, so by Theorem 8.4, $D(g) \cong 1_{N}$. As in the proof of Theorem 8.4, the trace of an isotopy from $D(g)$ to $1_{N}$ relative to $\partial N$ at each component $G$ of $\partial M-W$ lies in $G$, so by Theorem 4.2 we may deform the isotopy so that it preserves $\partial M-W$. Therefore $g$ is isotopic to $1_{M}$ relative to $W$ and hence relative to $F$.

Using Corollary 7.10, we have the following immediate consequence.

Theorem 8.6. Let $M$ be a Haken 3-manifold with incompressible boundary, and let $F$ be a nonempty compact 2-manifold in $\partial M$, such that $\partial M-F$ is incompressible. Then $\mathcal{H}(M$ rel $F)$ is geometrically finite.

From this we will obtain the following generalized version of the Kontsevich Conjecture for Haken manifolds.

Theorem 8.7. Let $M$ be a Haken 3-manifold with incompressible boundary, and let $F$ be a nonempty compact 2-manifold in $\partial M$ such that $\partial M-F$ is incompressible. Then $\operatorname{BDiff}(M$ rel $F)$ has the homotopy type of a finite complex.

Proof. We will show that $\pi_{i}(\operatorname{Diff}(M$ rel $F))=0$ for $i \geq 1$. Since $\operatorname{BDiff}(M \operatorname{rel} F)$ is connected and $\pi_{i+1}(\operatorname{BDiff}(M \operatorname{rel} F)) \cong \pi_{i}(\operatorname{Diff}(M \operatorname{rel} F))$ for $i \geq 1$, this implies that $\operatorname{BDiff}(M$ rel $F)$ is a $K(\mathcal{H}(M$ rel $F), 1)$-complex, 
so Theorem 8.6 shows that $\operatorname{BDiff}(M$ rel $F$ ) has the homotopy type of a finite complex.

By the main theorem of [12], the homotopy groups $\pi_{i}(\operatorname{Diff}(M \operatorname{rel} \partial M))$ vanish for $i \geq 1$, which gives the assertion when $F=\partial M$. Otherwise, let $W=\overline{\partial M-F}$. Restricting diffeomorphisms to $W$ is the projection map of a fibration

$$
\operatorname{Diff}(M \operatorname{rel} \partial M) \rightarrow \operatorname{Diff}(M \text { rel } F) \rightarrow \operatorname{Diff}(W \text { rel } \partial W) .
$$

From the homotopy exact sequence of this fibration, we have using [12] again, that $\pi_{i}(\operatorname{Diff}(M \operatorname{rel} F)) \cong \pi_{i}(\operatorname{Diff}(W \operatorname{rel} \partial W))=0$ for $i \geq 2$. We also obtain an exact sequence

$$
0 \rightarrow \pi_{1}(\operatorname{Diff}(M \operatorname{rel} F)) \rightarrow \pi_{1}(\operatorname{Diff}(W \operatorname{rel} \partial W)) \rightarrow \mathcal{H}(M \operatorname{rel} \partial M) .
$$

No component of $W$ is a 2-sphere, so elements in $\pi_{1}(\operatorname{Diff}(W$ rel $\partial W))$ are classified by their traces (nontrivial elements occur only for tori). Since the traces of an isotopy from $1_{M}$ to $1_{M}$ at different basepoints are freely homotopic, and $F$ is nonempty, all traces of an element of $\pi_{1}(\operatorname{Diff}(M \operatorname{rel} F))$ must be trivial in $\pi_{1}(M)$. Since $W$ is incompressible, the restriction of an element of $\pi_{1}(\operatorname{Diff}(M$ rel $F))$ to $W$ has trivial trace in each component of $W$, so is trivial in $\pi_{1}(\operatorname{Diff}(W$ rel $\partial W))$. Therefore $\pi_{1}(\operatorname{Diff}(M$ rel $F))$ is trivial.

\section{References}

[1] A. Borel and J.-P. Serre, Corners and arithmetic groups, Comment. Math. Helv., 48 (1973), 436-491.

[2] R. Canary and D. McCullough, Homotopy equivalences of 3-manifolds and deformation theory of Kleinian groups, in preparation.

[3] L.S. Charlap and A.T. Vasquez, Compact flat Riemannian manifolds III; the group of affinities, Amer. J. Math., 95 (1973), 471-494.

[4] H. Farkas and I. Kra, Riemann Surfaces, Springer-Verlag Graduate Texts in Mathematics $71,1980$.

[5] A. Fathi, F. Laudenbach and V. Poenaru, Travaux de Thurston sur les surfaces, Astérisque, 66-67 (1979).

[6] A. Gramain, Le type d'homotopie du groupe des difféomorphismes d'une surface compacte, Ann. scient. Éc. Norm. Sup., 6(4) (1973), 53-66.

[7] W. Hantzsche and W. Wendt, Drei dimensionale Euklidische Raumformen, Math. Ann., 110 (1934), 593-611.

[8] J. Harer, The virtual cohomological dimension of the mapping class group of an orientable surface, Invent. Math., 84 (1986), 157-176.

[9] The cohomology of the moduli space of curves, in 'Theory of Moduli', ed. E. Sernesi, Springer-Verlag Lecture Notes in Mathematics, 1337 (1988), 138-221.

[10] W. Harvey, Geometric structure of surface mapping class groups, in 'Homological Group Theory', ed. C.T.C. Wall, London Math. Soc. Lecture Note Series, 36 (1979), 255-269. 
[11] , Boundary structure of the modular group, in 'Riemann Surfaces and Related Topics: Proceedings of the 1978 Stony Brook Conference', ed. I. Kra and B. Maskit, Annals of Math. Study, 97 (1981), 245-251.

[12] A. Hatcher, Homeomorphisms of sufficiently large $P^{2}$-irreducible 3-manifolds, Topology, 15 (1976), 343-347.

[13] _ On triangulations of surfaces, Topology Appl., 40 (1991), 189-194.

[14] A. Hatcher and D. McCullough, Finiteness of classifying spaces of relative diffeomorphism groups of Haken 3-manifolds, Geometry and Topology, 1 (1997), 91-109.

[15] W. Heil and J. Tollefson, On Nielsen's theorem for 3-manifolds, Yokohama Math. J., 35 (1987), 1-20.

[16] W. Jaco and P. Shalen, Seifert fibered spaces in 3-manifolds, Mem. Amer. Math. Soc., 220 (1979), 1-192.

[17] K. Johannson, Homotopy Equivalences of 3-manifolds with Boundary, Springer-Verlag Lecture Notes in Mathematics, 761 (1979).

[18] Y. Kamishima, K.B. Lee and F. Raymond, The Seifert construction and its application to infranilmanifolds, Quart. J. Math., 34 (1983) 433-452.

[19] A. Karrass, A. Pietrowski and D. Solitar, Finite and infinite cyclic extensions of free groups, J. Australian Math. Soc., 16 (1972), 458-466.

[20] R. Kirby, Problems in low-dimensional topology, in 'Geometric Topology Part II', ed. W. Kazez, AMS/IP Studies in Advanced Mathematics, 2.2 (1997), 35-473.

[21] J.S. Lee, Almost periodic homeomorphisms and p-adic transformation groups on compact 3-manifolds, Proc. Amer. Math. Soc., 121 (1994), 267-273.

[22] F. Laudenbach, Topologie de dimension trois. Homotopie et isotopie, Astríisque, 12 (1974), 11-152.

[23] R. Lickorish, Homeomorphisms of non-orientable two-manifolds, Proc. Cambridge Phil. Soc., 59 (1963), 307-317.

[24] G.S. McCarty, Homeotopy groups, Trans. Amer. Math. Soc., 106 (1963), 293-304.

[25] D. McCullough, Mappings of reducible 3-manifolds, in 'Proceedings of the Semester on Topology of the Stefan Banach International Mathematical Center', ed. H. Toruńczyk, Banach Center Publications, Warsaw, (1986), 61-76.

[26] _ Virtually geometrically finite mapping class groups of 3-manifolds, J. Diff. Geom., 33 (1991), 1-65.

[27] _ 3-Manifolds And Their Mappings, Global Analysis Center Lecture Note Series 26, Seoul National University, 1995.

[28] D. McCullough and A. Miller, Symmetric automorphisms of free products, Mem. Amer. Math. Soc., 582 (1996), 1-97.

[29] P. Orlik, Seifert Manifolds, Springer-Verlag Lecture Notes in Mathematics, 291 (1972).

[30] H. Schneebeli, On virtual properties and group extensions, Math. Z., 159 (1978), 159-167.

[31] P. Scott, The geometries of 3-manifolds, Bull. London Math. Soc., 15 (1983), 401-487.

[32] J. van Mill, Infinite-Dimensional Topology, Prerequisites and Introduction, NorthHolland Mathematical Library, 43 (1989). 
[33] F. Waldhausen, Eine Klasse von 3-dimensionalen Mannigfaltigkeiten I, II, Invent. Math., 3 (1967), 308-333; 4(1967), 87-117.

[34] _ On irreducible 3-manifolds which are sufficiently large, Ann. of Math., 87 (1968), 56-88.

[35] J. West, Mapping Hilbert manifolds to ANR's: A solution of a conjecture of Borsuk, Ann. of Math., 106(2) (1977), 1-18.

[36] B. Zimmermann, On the Hantzsche-Wendt manifold, Monatsh. Math., 110 (1990), 321-327.

Received July 14, 1997.

Korea University

SEOUL 136-701

KoreA

E-mail address: shong@semi.korea.ac.kr

UNIVERSITY OF OKLAHOMA

NORMAN, OK 73019

E-mail address: dmccullough@math.ou.edu 